\title{
Reasons Patients With a Positive Fecal Occult Blood Test Result Do Not Undergo Complete Diagnostic Evaluation
}

\author{
Masabito Jimbo, $M D, P b D, M P H^{1}$ \\ Ronald E. Myers, $P b D^{2}$ \\ Birgit Meyer, $M D^{3}$ \\ Terry Hyslop, PbD ${ }^{4}$ \\ James Cocroft, $M S^{2}$ \\ Barbara J. Turner, $M D^{5}$ \\ David S. Weinberg, MD \\ 'Department of Family Medicine, Univer- \\ sity of Michigan, Ann Arbor, Michigan \\ ${ }^{2}$ Department of Medical Oncology, \\ Thomas Jefferson University, Philadelphia, \\ Pennsylvania \\ ${ }^{3}$ Doernbecher Children's Hospital, Port- \\ land, Oregon \\ ${ }^{4}$ Division of Biostatistics, Department of \\ Pharmacology and Experimental Therapeu- \\ tics, Thomas Jefferson University, Philadel- \\ phia, Pennsylvania \\ ${ }^{5}$ Department of Medicine, University of \\ Pennsylvania, Philadelphia, Pennsylvania \\ ${ }^{6}$ Department of Medical Oncology, Fox \\ Chase Cancer Center, Philadelphia, \\ Pennsylvania

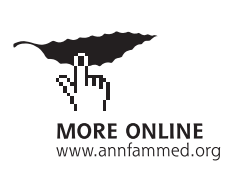

Conflicts of interest: none reported

\section{CORRESPONDING AUTHOR}

Masahito Jimbo, MD, PhD, MPH 1018 Fuller Street, SPC 5708 Ann Arbor, MI 48109-5708

mjimbo@med.umich.edu

\begin{abstract}
PURPOSE Screening for fecal occult blood reduces colorectal cancer mortality by identifying patients with positive results for complete diagnostic evaluation (CDE). CDE rates are suboptimal, however. We sought to determine common reasons for nonperformance of a CDE as recorded by the primary care physician.
\end{abstract}

METHODS We undertook a descriptive analysis of reasons reported by physicians for nonperformance of $C D E$ in a nested sample of patients with positive fecal occult blood test (FOBT) results from a randomized controlled trial designed to evaluate the impact of a physician intervention (CDE reminder-feedback and educational outreach) on recommendation and performance rates in primary care practices. Inspection of administrative data for 1,468 patients with positive results showed that 661 (45\%) did not undergo CDE. We reviewed patient followup forms, which were completed by physicians for patients who did not have a $C D E$, to identify reasons for nonperformance.

RESULTS Nonperformance of CDE was due to physician decision for 217 patients (33\%). In 123 patients (19\%), reasons for nonperformance were compatible with the guidelines, and in 94 patients (14\%), they were not. Reasons wholly or partially due to factors other than physician decision were noted in 212 patients (32\%); physician action was considered to be appropriate in these patients. For the 232 patients (35\%) without a clearly documented reason for CDE nonperformance, the appropriateness of the physicians' action could not be determined.

CONCLUSIONS Decision making by primary care physicians had a major effect on nonperformance of CDE after a positive FOBT result. Colorectal cancer screening programs should include guidance for physicians about when a CDE should and should not be performed.

Ann Fam Med 2009;7:11-16. DOI: 10.1370/afm.906.

\section{INTRODUCTION}

$\Lambda$ nnual fecal occult blood test (FOBT) screening is endorsed by major medical organizations as a viable colorectal cancer screening strategy. ${ }^{1,2}$ Large, randomized controlled trials have shown that screening for colorectal cancer by FOBT can significantly lower incidence and mortality. ${ }^{3-5}$ In these trials, more than $80 \%$ of patients with positive FOBT results underwent a complete diagnostic evaluation (CDE), which consists of either colonoscopy or double-contrast barium enema plus flexible sigmoidoscopy. Reductions in mortality observed in these trials resulted from the detection and treatment of asymptomatic, early-stage colorectal cancer and colorectal adenomas.

Unfortunately, in clinical practice rates of CDE are low for patients whose test results were positive for fecal occult blood, ranging from $39 \%$ to $59 \% .^{6-16}$ These data suggest that opportunities to diagnose early colorectal cancer and colorectal polyps may frequently be missed among 
patients who have positive FOBT results. Little is currently known, however, about the specific reasons for nonperformance of a complete evaluation. In this study, we analyzed physician-reported reasons for nonperformance of CDE for patients who had positive FOBT results during a 13 -month period of a randomized trial designed to evaluate the impact of a physician intervention on recommendation and performance rates in primary care practices.

\section{METHODS}

From 1997 to 2001, a research team from Thomas Jefferson University performed a study funded by the National Cancer Institute to evaluate the impact of a physician intervention on CDE recommendation and performance rates for positive FOBT results in primary care practices. ${ }^{17-19}$ Eligible practices had patients insured with a major managed care organization (MCO) in southeastern Pennsylvania and southern New Jersey, and they had 3 or more patients with positive FOBT results within the previous 18 months. Of the 584 eligible practices, 431 agreed to participate, and 318 returned at least 1 physician baseline survey form. These practices were randomly assigned either to a control group $(\mathrm{n}=198)$ or to an intervention group $(\mathrm{n}=120)$. The physicians in the intervention group received CDE reminder-feedback plus educational outreach. The study was approved by the Institutional Review Board at Thomas Jefferson University. Written consent for participation was obtained from study physicians in accordance to the directions provided by the institutional review board.

Patients in the control and intervention group practices routinely received standard colorectal cancer screening sponsored by the MCO. MCO subscribers aged 50 to 74 years were mailed an annual FOBT kit that contained an introductory letter, information about colorectal cancer, 3 Hemoccult II slides (Beckman Coulter, Fullerton, California) with instructions, and a postage-paid return envelope, as well as printed instructions regarding diet and medication restrictions during the testing period. FOBT cards were returned to the Thomas Jefferson University Hospital Clinical Laboratory for analysis. The laboratory notified the patient and the patient's primary care practice of the test results.

For patients with positive FOBT results, the research team sent patient-specific internal chart audit forms to the patient's practice for follow-up information. Control group practices received the audit forms at the conclusion of the study; intervention group practices received the form during the study as part of the reminder-feedback component of the study inter- vention. Physicians were asked to indicate whether they had advised and performed a CDE for patients who had positive results within 180 days after the positive result date, and if performed, the procedure date. Audit forms were linked to the practice but did not link the physician to the patient. ${ }^{19}$

We determined the recommendation and performance status for each patient by combining data from the internal audit forms with data from the MCO administrative claims for colonoscopy. This method has high sensitivity and specificity when compared with external chart audit and administrative data review. ${ }^{18}$ If a CDE was not performed, space was provided on the form where the physician could list up to 3 reasons for nonperformance. A sample audit form is displayed in the Supplemental Appendix, available online at http://www.annfammed. org/cgi/content/full/7/1/11/DC1.

From the survey data we captured physician age, specialty, board certification status, sex, ethnicity, and years in practice. These data were aggregated by practice to generate practice-level characteristics and to compare them with practice-level data for reasons for evaluation nonperformance. ${ }^{19}$

Reasons reported on the audit forms for nonperformance were transcribed verbatim for each practice. Three coauthors (R.E.M., B.J.T., D.S.W.) performed content analysis. Initial coding categories were developed through an iterative review of the forms. Any disagreements in category assignment among reviewers were resolved through discussions that led to consensus. Each coding category was placed in 1 of 3 groups: physician decision, nonphysician factors, or no documented reasons.

For physician decision, the coding categories reflected the physician's explicit decision not to pursue a CDE. Each category was checked for compatibility with the current guidelines. ${ }^{20-25}$ Two categories were compatible with guidelines: CDE completion within 3 years before a positive FOBT result, and medical contraindication. The other categories were not compatible with guidelines: normal findings from non-CDE procedure, such as a repeat FOBT, sigmoidoscopy, or upper gastrointestinal radiography; non-CDE procedure before the positive FOBT results within 3 years before a positive FOBT result; use of medications, medical history, and nonadherence to diet, which explained the positive FOBT result; fewer than 3 positive FOBT results cards; no family history; and old age

For nonphysician factors, nonperformance was not due to the physician's decision: patient referred but did not undergo CDE; and patient refused, patient deceased, and patient left practice. Some factors were practice-specific: no FOBT result record in the prac- 
tice $_{\text {; }}$ and patient not known by the practice at the time of the positive FOBT result. Other factors included reasons not attributable to any of the above, eg, other reason not otherwise specified.

For patients without clearly documented reasons, the explicit reason for CDE nonperformance could not be ascertained: "no documentation" of any reason for CDE nonperformance; "no information" justifying CDE nonperformance; and "no rationale" for CDE nonperformance.

\section{RESULTS}

From January 1999 to January 2000, the central FOBT testing laboratory identified 1,508 patients with positive results in the $318 \mathrm{MCO}$ screening program study practices that were initially considered for inclusion in data analysis; $61 \%$ were men and 39\% were women. Internal chart audit forms and administrative data were obtained for $1,468(97 \%)$ of the patients with positive results; 807 (55\%) underwent CDE, and 661 patients (45\%) did not (Figure 1).

The 201 practices with the 661 patients for whom CDE was not performed made up our study group. Characteristics of the study practices are displayed in Table 1. Larger family practices predominated. When compared with the 318 practices in the larger trial, the 201 study practices were similar in that most physicians in the practices were male, white, and board-certified, and they tended to be older and more experienced. Three hundred eighty-three (58\%) patients were in the in the control group practices, and 278 (42\%) patients were in the intervention group practices of the larger trial.

Of the 661 patients in our study group practices, 404 (61\%) were men, 257 (39\%) were women, 202 (33\%) were aged 50 to 64 years, and 422 (67\%) were aged 65 years and older. (For 37 patients age data were missing.) Characteristics for the study group patients were not significantly different from those of the overall patients who had positive FOBT results. For 429 of the 661 patients $(65 \%)$, the physician reported 1 or more reasons attributable to a physician decision, nonphysician factors, or both for CDE nonperformance. The remaining 232 patients (35\%) did not have a clearly documented reason (Table 2 ).

For each patient, we determined whether the action taken by the physician was appropriate. For patients with 1 or more physician-decision reasons only, if at least 1 reason for CDE nonperformance was compatible with the guidelines, we concluded that the physician took appropriate action. If no reason for the physician decision was compatible with the guidelines, we concluded that the physician took inappropriate action.

\section{Figure 1. Study flow diagram.}

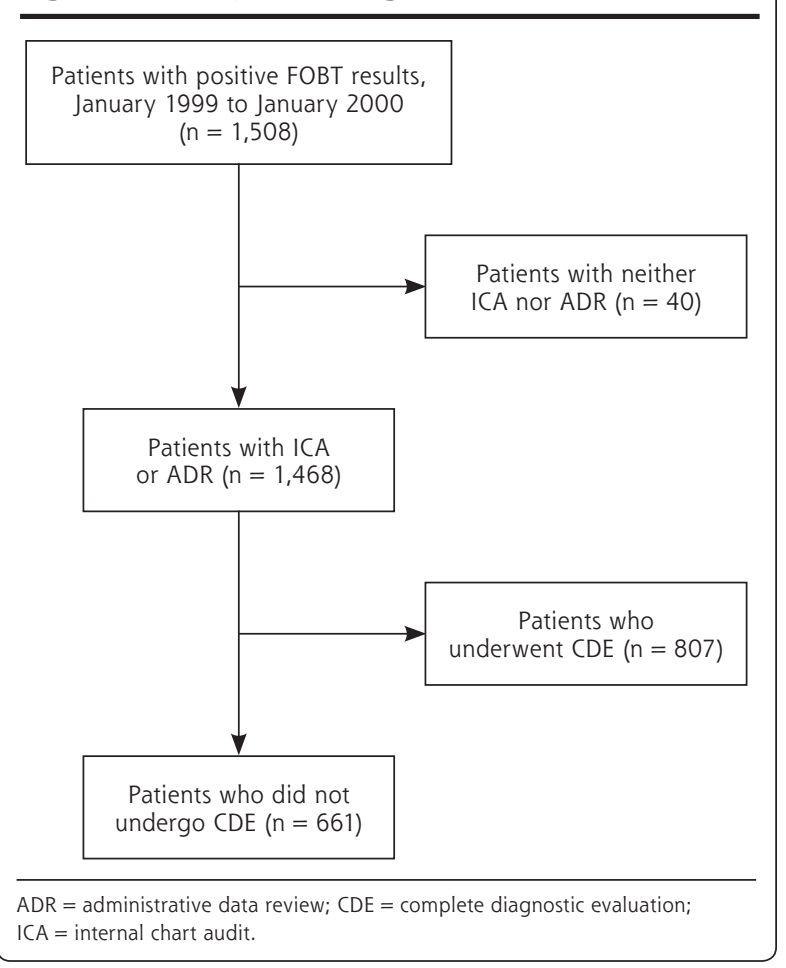

Table 1. Characteristics of Practices With Patients Who Had Positive Fecal Occult Blood Test Results but No Complete Diagnostic Evaluation ( $N=201)$

\begin{tabular}{lc}
\hline Characteristic & $\mathbf{n}(\%)$ \\
\hline Administrative arrangement & $53(26)$ \\
Solo & $148(74)$ \\
Group & \\
Physician age (median) & $90(45)$ \\
Less than 45 years & $109(55)$ \\
45 years or older & \\
Specialty & $146(73)$ \\
Family medicine & $44(22)$ \\
Internal medicine & $11(5)$ \\
Both specialties & $154(77)$ \\
Board certified & $47(23)$ \\
$100 \%$ & $133(66)$ \\
Not 100\% & $68(34)$ \\
Sex & \\
All male & $163(81)$ \\
Not all male & $38(19)$ \\
Ethnicity & $91(45)$ \\
All white & $109(55)$ \\
Not all white & \\
Years in practice (median) & \\
$<18$ y & \\
$\geq 18$ y & Note: Not all practices provided complete demographic information. Missing \\
numbers are from solo practices. General practice made up just $8 \%$, or $4 \%$ of \\
the practices, and was combined with family medicine.
\end{tabular}




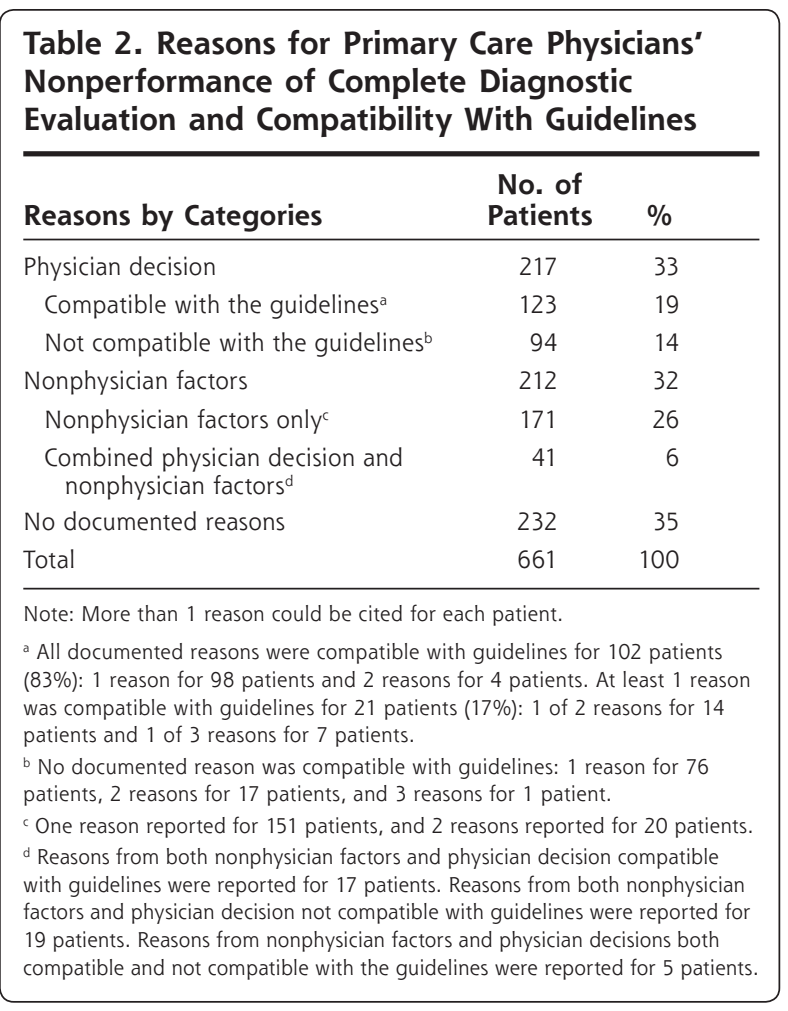

For patients with 1 or more nonphysican-factor reasons (including nonphysician factors only and both nonphysician factors and physician decision), we concluded that CDE nonperformance was not due to the physician's decision and that the physician took appropriate action. For patients with no documented reason for nonperformance, we concluded that the appropriateness of the physician's action could not be determined.

There were 217 patients with positive FOBT results for whom physicians had chosen not to perform further evaluation, 123 (57\%) of whom had appropriate action taken. Reasons compatible with guidelines included CDE completion within 3 years (97) and medical contraindication (30). The remaining 94 patients (43\%) had inappropriate action taken, with the most cited reason being normal findings from non-CDE procedure (69). The rest of the reasons were distributed among medical history (15), use of medications (13), non-CDE procedure befor the positive FOBT result (10), nonadherence to diet (3), fewer than 3 positive FOBT results (1), no family history (1), and old age (1).

There were 212 patients for whom nonperformance was due to nonphysician factors. For 171 (81\%) of these patients, physicians cited nonphysician factors only, of which the most common were patient referred (79), and patient refused (55). The rest were distributed among no FOBT record (19), other factors (11), patient left practice (10), patient deceased (9), and patient not known (8). For the other 41 patients, rea- sons came from both nonphysician factors and physician decisions.

There were 232 patients without a clearly documented reason for nonperformance. These reasons included no documentation (159), no information (44), and no rationale (29).

\section{DISCUSSION}

In this study, the physician's reported reason for CDE nonperformance was appropriate for 335 (51\%) of 661 patients, or $78 \%$ of those patients with clearly documented reasons. Physician decision was the key factor in a slight majority of patients with clearly documented reasons. A common reason given by the physicians for not performing a CDE was that a diagnostic evaluation was completed within 3 years before the positive FOBT result. That patients with positive results had a recent CDE is likely, because the MCO screening program was directed toward all patients aged 50 to 74 years. It is plausible that patients who had recently undergone diagnostic workup were included in the population screened. The physician's decision not to pursue a CDE with these patients is appropriate, given that guidelines recommend deferring colorectal cancer screening for 5 years after finding negative results on a CDE, and FOBT is not recommended for patients under surveillance. ${ }^{20,21}$ General mailings of FOBT cards from the $\mathrm{MCO}$ without considering a history of CDE may increase unnecessary colonoscopy use; the physician serves an important role in avoiding such overutilization. Medical conditions that contraindicated a CDE are also compatible with the guidelines.

On the other hand, other physician-decision reasons were not compatible with guidelines. Non-CDE procedures, such as flexible sigmoidoscopy, are not considered adequate substitutes for a CDE. ${ }^{22}$ In addition, a patient's medical history that suggests a possible explanation for having a positive result, patient age, and lack of family history are not considered to be appropriate reasons to forego $\mathrm{CDE}$. It is important to note that there is no agreed-upon number of positive FOBT cards needed to determine a patient has positive result. Thus, deciding not to perform CDE for patients with positive FOBT results unless all of their 3 cards tested positive is unsupported by the guidelines. ${ }^{10}$ Finally, despite concerns regarding their possible effect on FOBT, dietary noncompliance or the use of contraindicated medications would not justify CDE nonperformance. ${ }^{23-25}$

In determining the appropriateness of physician action for CDE nonperformance, we concluded that an inappropriate action was taken with only those patients for whom none of the reported reasons was compatible with the guidelines. We could not determine the 
appropriateness for $35 \%$ of the patients who had no clearly documented reason for CDE nonperformance. For patients with no documentation, the physician may have taken appropriate action and documented it in the chart but not in the audit; the physician may have taken appropriate action but not documented it in the chart or audit; or the physician may have taken inappropriate action and not documented it in the audit. The latter 2 possibilities would also apply to patients for whom the physician could not find the information justifying CDE nonperformance and to patients for whom the physician admitted that there was no clear rationale for nonperformance. For some patients one or more reasons for nonperformance were due to physician decisions or to a combination of nonphysician factors and physician decisions that were not compatible with the guidelines. The latter included patient referred or patient refused further evaluation. Physicians who believed their patients had reason not to pursue a CDE may be less inclined to aggressively follow through on patient referrals or recommend their patients for a CDE.

Reductions in colorectal cancer incidence and mortality reported in large, well-designed, randomized controlled trials were contingent on appropriate follow-up of patients with positive FOBT results who had had a CDE. ${ }^{3-5}$ There is little evidence that such levels of evaluation have been achieved in routine care. On reviewing 11 studies reported from 1991 through 2006, we found that CDE rates in clinical practice have been suboptimal, ranging from $39 \%$ to $59 \%$, and there does not appear to be a trend showing increased CDE performance over time. ${ }^{6-16}$ In 2005 Nadel et al reported that $30 \%$ of the physicians in a national survey recommended another FOBT as a follow-up test for earlier positive results. ${ }^{13}$ In a more recent study, Fisher et al reported that $20 \%$ of patients in a single Veterans Affairs center did not keep the scheduled consultant appointment for a CDE..$^{15}$ These studies have not looked at the specific reasons for CDE nonperformance. The study reported here addresses the need for information on this issue.

In an earlier report on physician-reported reasons for CDE nonperformance in patients with positive results, we included practices and physicians before randomization in the trial. ${ }^{26}$ That report included data collected from physician reports on a subset of a small number of patients $(n=248)$ for an abbreviated observation period, whereas the current study involved data collection for 661 patients over a 13-month observation period. In this study we also analyzed the appropriateness of CDE nonperformance for all patients, whereas in the early report we did not. We believe the current report provides a more definitive assessment and more complete ascertainment of factors that accounted for CDE nonperformance.
Of note, there were more practices in the control group than in the intervention group in the larger randomized trial. Furthermore, exposure of intervention group physicians to the CDE reminder-feedback and educational outreach intervention served to increase practice recommendation and performance rates significantly. ${ }^{17}$ Patients with positive FOBT results seen in intervention group practices were thus more likely to undergo a CDE than were patients with positive results in control group practices. As a consequence, patients with positive FOBT results in the current study were more likely to be drawn from control group practices. Given this situation, we believe that any bias created by exposure to the physician intervention used in the randomized trial is likely to be minimal. Additionally, because the physicians in the control group practices received the audit forms at the conclusion of the study, they would have been more likely than those in intervention grouop practices to have information on FOBT follow-up and CDE at the time of the audit.

Although the national trend shows a gradual increase in the use of colonoscopy and a decrease in the use of FOBT in colorectal cancer screening, the latter remains an important option. ${ }^{27}$ Currently, sufficient capacity may not exist to provide colonoscopy as the only screening option to all eligible patients. There already exists sufficient capacity for annual FOBT with a CDE for positive results, arguing for continued use of FOBT as a viable colorectal cancer screening option. ${ }^{28}$

In its recently published monograph, ${ }^{29}$ the National Cancer Institute set a priority to "determine why abnormal findings from screening examinations have less than acceptable rates of follow-up and develop strategies to improve the system." Our study directly addresses this priority. By elucidating the reasons for nonperformance of CDEs, findings from this study could guide future research to develop specific interventions to improve follow-up of abnormal colorectal cancer screening results. Our findings suggest that physician education in the appropriate indications for a CDE may warrant more attention.

The current study has several limitations in addition to those already mentioned. First, the study targeted primary care physicians only and did not include consultants. Thus, CDE nonperformance for patients referred may actually be due to a consultant decision (eg, the consultant believed that CDE was not necessary), patient decision (eg, patient did not follow through with the referral), or practice factors (eg, the practice did not generate a referral for the patient). Second, our definition of CDE included double-contrast barium enema plus flexible sigmoidoscopy as well as colonscopy. Recent guidelines by the US Multisociety Task Force on colorectal cancer recommend 
colonoscopy as the preferred diagnostic procedure. ${ }^{22}$ Even so, double-contrast barium enema plus flexible sigmoidoscopy is still considered to be an acceptable alternative if colonoscopy is not available, not feasible, or not desired by the patient. ${ }^{22}$

Reasons for nonperformance of a CDE are multifactorial. Although most physicians had appropriate reasons for nonperformance, further analysis of the reasons showed that at least $14 \%$ of physicians' decisions not to perform a CDE was not compatible with the guidelines. Findings reported here will shed light on potential interventions targeting physicians to improve further evaluation for patients with positive FOBT results.

To read or post commentaries in response to this article, see it online at http://www.annfammed.org/cgi/content/full/7/1/111.

Submitted November 6, 2007; submitted, revised, May 26, 2008; accepted June 13, 2008.

Key words: Colorectal neoplasm; occult blood/fecal; mass screening; follow-up studies; FOBT

This study was previously presented as an oral presentation by Jimbo Myers, Hyslop, and Cocroft, at the 35th Annual Meeting of the North American Primary Care Research Group, October 2007, Vancouver, British Columbia, Canada.

Funding support: This study was supported by the National Cancer Institute (grant 5R01 CA68683) and Aetna US Healthcare, which now operates as Aetna, Inc.

\section{References}

1. Levin B, Lieberman DA, McFarland B, et al. Screening and surveillance for the early detection of colorectal cancer and adenomatous polyps, 2008: a joint guideline from the American Cancer Society, the US Multi-Society Task Force on Colorectal Cancer, and the American College of Radiology. CA Cancer J Clin. 2008;58(3):130-160.

2. US Preventive Services Task Force. Screening for colorectal cancer: recommendation and rationale. Ann Intern Med. 2002;137(2):129-141.

3. Hardcastle JD, Chamberlain JO, Robinson MH, et al. Randomised controlled trial of faecal-occult-blood screening for colorectal cancer. Lancet. 1996;348(9040):1472-1477.

4. Kronborg O, Fenger C, Olsen J, Jorgensen OD, Sondergaard O. Randomised study of screening for colorectal cancer with faecal-occultblood test. Lancet. 1996;348(9040):1467-1471.

5. Mandel JS, Bond JH, Church TR, et al. Reducing mortality from colorectal cancer by screening for fecal occult blood. Minnesota Colon Cancer Control Study. N Engl J Med. 1993;328(19):1365-1371.

6. Morris JB, Stellato TA, Guy BB, Gordon NH, Berger NA. A critical analysis of the largest reported mass fecal occult blood screening program in the United States. Am J Surg. 1991;161(1):101-105. [discussion 105-106].

7. Myers RE, Balshem AM, Wolf TA, Ross EA, Millner L. Screening for colorectal neoplasia: physicians' adherence to complete diagnostic evaluation. Am J Public Health. 1993;83(11):1620-1622.

8. Levin B, Hess K, Johnson C. Colorectal cancer screening in the community: adequacy of follow-up is related to physician specialty. Gastroenterology. 1996;110:A550.
9. Lurie JD, Welch HG. Diagnostic testing following fecal occult blood screening in the elderly. J Natl Cancer Inst. 1999;91(19):1641-1646.

10. Sharma VK, Vasudeva R, Howden CW. Colorectal cancer screening and surveillance practices by primary care physicians: results of a national survey. Am J Gastroenterol. 2000;95(6):1551-1556.

11. Shields HM, Weiner MS, Henry DR, et al. Factors that influence the decision to do an adequate evaluation of a patient with a positive stool for occult blood. Am J Gastroenterol. 2001;96(1):196-203.

12. Ko CW, Dominitz JA, Nguyen TD. Fecal occult blood testing in a general medical clinic: comparison between guaiac-based and immunochemical-based tests. Am J Med. 2003;115(2):111-114.

13. Nadel MR, Shapiro JA, Klabunde CN, et al. A national survey of primary care physicians' methods for screening for occult blood. Ann Intern Med. 2005;142(2):86-94.

14. Etzioni DA, Yano EM, Rubenstein LV, et al. Measuring the quality of colorectal cancer screening: the importance of follow-up. Dis Colon Rectum. 2006;49(7):1002-1010.

15. Fisher DA, Jeffreys A, Coffman CJ, Fasanella K. Barriers to full colon evaluation for a positive fecal occult blood test. Cancer Epidemiol Biomarkers Prev. 2006;15(6):1232-1235.

16. Garman KS, Jeffreys A, Coffman C, Fisher DA. Colorectal cancer screening, comorbidity, and follow-up in elderly patients. Am J Med Sci. 2006;332(4):159-163.

17. Myers RE, Turner B, Weinberg D, et al. Impact of a physician-oriented intervention on follow-up in colorectal cancer screening. Prev Med. 2004;38(4):375-381.

18. Myers RE, Fishbein G, Hyslop T, et al. Measuring complete diagnostic evaluation in colorectal cancer screening. Cancer Detect Prev. 2001;25(2):174-182.

19. Myers RE, Turner B, Weinberg D, et al. Complete diagnostic evaluation in colorectal cancer screening: research design and baseline findings. Prev Med. 2001;33(4):249-260.

20. American College of Physicians. Clinical guideline: part I: suggested technique for fecal occult blood testing and interpretation in colorectal cancer screening. Ann Intern Med. 1997;126(10):808-810.

21. Winawer SJ, Zauber AG, Fletcher RH, et al. Guidelines for colonoscopy surveillance after polypectomy: a consensus update by the US Multi-society Task Force on Colorectal Cancer and the American Cancer Society. Gastroenterology. 2006;130(6):1872-1885.

22. Winawer $S$, Fletcher R, Rex D, et al. Colorectal cancer screening and surveillance: clinical guidelines and rationale-update based on new evidence. Gastroenterology. 2003;124(2):544-560.

23. Macrae FA, St John DJ, Caligiore P, Taylor LS, Legge JW. Optimal dietary conditions for hemoccult testing. Gastroenterology. 1982;82(5 Pt 1):899-903.

24. Pye G, Ballantyne KC, Armitage NC, Hardcastle JD. Influence of non-steroidal anti-inflammatory drugs on the outcome of faecal occult blood tests in screening for colorectal cancer. Br Med J (Clin Res Ed). 1987;294(6586):1510-1511.

25. Greenberg PD, Cello JP, Rockey DC. Asymptomatic chronic gastrointestinal blood loss in patients taking aspirin or warfarin for cardiovascular disease. Am J Med. 1996;100(6):598-604.

26. Baig N, Myers RE, Turner BJ, et al. Physician-reported reasons for limited follow-up of patients with a positive fecal occult blood test screening result. Am J Gastroenterol. 2003;98(9):2078-2081.

27. Seef LC, King J, Pollack A, Williams KN. Increased use of colorectal cancer tests-United States, 2002 and 2004. MMWR Morb Mortal Wkly Rep. 2006;55(11):308-311.

28. Seeff LC, Manninen DL, Dong FB, et al. Is there endoscopic capacity to provide colorectal cancer screening to the unscreened population in the United States? Gastroenterology. 2004;127(6):1661-1669.

29. National Cancer Institute. The NCI Strategic Plan For Leading the Nation to Eliminate the Suffering and Death Due to Cancer. Bethesda, MD: National Cancer Institute; 2006. 